\title{
A user friendly software for the design of full bridge reduced models in wind tunnel testing
}

\author{
M. Cid Montoya ${ }^{1}$, S. Hernández ${ }^{1}$, F. Nieto ${ }^{1}$, J. P. C. King ${ }^{2}$ \\ \& L. Kong ${ }^{2}$ \\ ${ }^{1}$ School of Civil Engineering, Universidade da Coruña, Spain \\ ${ }^{2}$ The University of Western Ontario, Faculty of Engineering, Canada
}

\begin{abstract}
Wind tunnel aeroelastic tests of full bridge reduced models are nowadays a mandatory step in the design of long-span bridges. The tests require complex, timedemanding and expensive experimental campaigns which consequently increase the costs of the projects. These campaigns consist of a long list of tasks, which includes wind characterization, model design and construction, instrumentation calibration, data acquisition and post-processing of the acquired data, among others. Thus, advances in any of these activities will always represent a technical advance in terms of test performance and economical efficiency. This paper focuses on the model design, and a brief description of the whole process is presented. Besides, the design process was implemented in an integrated software and it is presented with an application example of a generic cable-stayed bridge deck. The software allows the design of the whole reduced model in a user friendly way and in a very short working time.

Keywords: aeroelasticity, structural dynamics, reduced-scale models, wind tunnel, optimization.
\end{abstract}

\section{Introduction}

Full bridge aeroelastic wind tunnel tests $[1,2]$ are nowadays a mandatory step in the design of long-span bridges [3]. As the length of the spans of suspension and cable-stayed bridges is steadily increasing over the years, the effects of wind over these structures is more relevant, and consequently designs are increasingly constrained by aeroelastic effects $[4,5]$. In these tests, accurate representations of the surrounding terrain [6], wind characteristics [7,8], and bridge geometry 
and mechanical properties are required to obtain meaningful results [9]. These requirements lead to a complex setting up of tests aiming at faithfully representing

the real phenomena. Some examples of full-bridge aeroelastic tests can be also found, for instance, in $[10,11]$. This turns these tests into an expensive and burdensome task that requires a considerable human work, which makes the aeroelastic tests nowadays a challenge. Consequently, advances in any of the several steps or tasks comprised by the tests are welcome.

One of the most challenging task is the accurate model design, which is described, for instance, in $[12,13]$. In this task, the actual geometry of every element of the real bridge has to be reproduced, as well as its mechanical properties. This leads to the design of a scaled structure with different material and dimensions that has to aeroelastically behave in the same way as the prototype. This work presents the development of an integrated software to design full bridge reduced models in an easy and fast way, taking into account all the requirements needed to obtain an accurate model of the prototype. Besides, the software is tested with an application example of a cable-stayed bridge deck cross-section.

\section{General full bridge model design rules and basis of the software}

Full bridge modeling for aeroelastic wind tunnel tests consists of designing and producing scaled models to reproduce the prototype aeroelastic responses. Test characteristics and model construction limitations govern the reduced model design rules [14]. First, the dimensions of the test chamber establish the adequate model geometric scale, which conditions all the design in terms of mechanical properties, testing and results interpretation. Second, this geometric scale reduction set limitations in the construction of the mock-up, forcing the model designer to assume simplifications such as represent variable stiffness properties along lengths of the prototype elements by means of uniform crosssections, or concentrate mass properties in particular points.

In the following section these rules are commented and their implementation in the software presented in this work, named the Model Design Tool (MODESTO), are also presented.

\subsection{Similitude requirements}

When aiming to reproduce the full scale response of a prototype by means of a reduced model, it is mandatory to take into account the similarity requirements or model laws to achieve a good agreement between the responses of the reduced model and the prototype. Geometric similarity is achieved by scaling the geometry of the prototype employing the length scale factor $\lambda_{L}$. This scale factor is established by the relation between the prototype and test chamber dimensions. Besides, other non-dimensional quantities have to be considered in order to keep in the same ratio the forces acting on a mass of air as it happens in full scale [15]. 
These forces are the inertia forces, the gravitational forces and the viscous forces. The combinations of the effects of these forces gives place to the following ratios:

- The ratio of the gravitational to the inertia forces is defined by the Froude number $\left(F r=g L / V^{2}\right)$ and establishes the relation between the flow velocity $V$, a representative dimension of the model $L$ and the acceleration due to gravity $g$.

- The ratio of the elastic forces of the bridge to inertia forces of the flow is given by the Cauchy number $\left(C a=E I \rho V^{2}\right)$, and is employed to define the scale factors for the material and section properties.

- The Reynolds number similarity $(R e=V L / \nu)$ establishes the relationship between the flow velocity $V$, a representative dimension of the model $L$ and the viscosity of the flow $\nu$. However, keeping this similarity is not practical and tests are usually carried out without enforcing it, given that bridge decks are commonly sharp-edged bodies, which makes them relatively insensitive to Reynolds number effects.

- The mass of the model with respect to the air in the wind tunnel is fixed by the density ratio $\left(\rho_{\text {model }} / \rho_{\text {air }}\right)$.

- The damping scale factor $\left(\zeta_{\text {model }} / \zeta_{\text {prototype }}\right)$ is important to be preserved because of its influence on resonant motions. However, the model is commonly designed to have as low damping value as possible.

Thus, following this relations, all the scale factor required for carrying out the model design can be defined as

$$
\begin{array}{llll}
\lambda_{L}=L_{m} / L_{p} & \lambda_{\rho}=\rho_{m} / \rho_{p} & \lambda_{V}=V_{m} / V_{p} & \lambda_{m}=\lambda_{\rho} / \lambda_{L}^{2} \\
\lambda_{M}=\lambda_{\rho} / \lambda_{L}^{3} & \lambda_{i}=\lambda_{m} / \lambda_{L}^{2} & \lambda_{I}=\lambda_{M} / \lambda_{L}^{2} & \lambda_{T}=\lambda_{L} / \lambda_{V} \\
\lambda_{a}=\lambda_{V} / \lambda_{T} & \lambda_{\zeta}=\zeta_{m} / \zeta_{p} & \lambda_{\mathrm{EI}}=\lambda_{V}^{2} \lambda_{L}^{4} & \lambda_{\mathrm{EA}}=\lambda_{V}^{2} \lambda_{L}^{2} \\
\lambda_{f}=\lambda_{V}^{2} / \lambda_{L} & \lambda_{F}=\lambda_{V}^{2} / \lambda_{L}^{2} & \lambda_{\mathrm{BM}}=\lambda_{V}^{2} / \lambda_{L}^{3} & \lambda_{\mathrm{CWE}}=\lambda_{V}^{2} / \lambda_{L}^{6}
\end{array}
$$

where $\lambda_{L}$ is the length scale factor, $L$ represent a characteristic dimension, the subindeces $m$ and $p$ indicates model or prototype, respectively, $\lambda_{\rho}$ is the scale factor of the density $\rho, V$ indicates velocity, $M$ is the mass while $m$ is the mass per unit of length, $I$ and $i$ represent the mass moment of inertia and its value per unit of length, $T$ is the time, $a$ represent acceleration, $\zeta$ is the damping, the elastic stiffness is represented as EI for the bending and EA for the axial stiffness, $F$ and $f$ represent the force and its value per unit of length, BM is the bending and torsional moment and CWE the warping stiffness.

\subsection{Basis of the software operation}

The design of full bridge reduced models is based on defining an equivalent structure which accomplishes all or most of the similitude requirements outlined before. With regards to the structural behavior, this means defining and equivalent reduced-scale structure for the prototype. As this kind of structures generally behave as a set of beam elements, the process of designing the model can be 
split into an independent design process for each 1D element that composes the

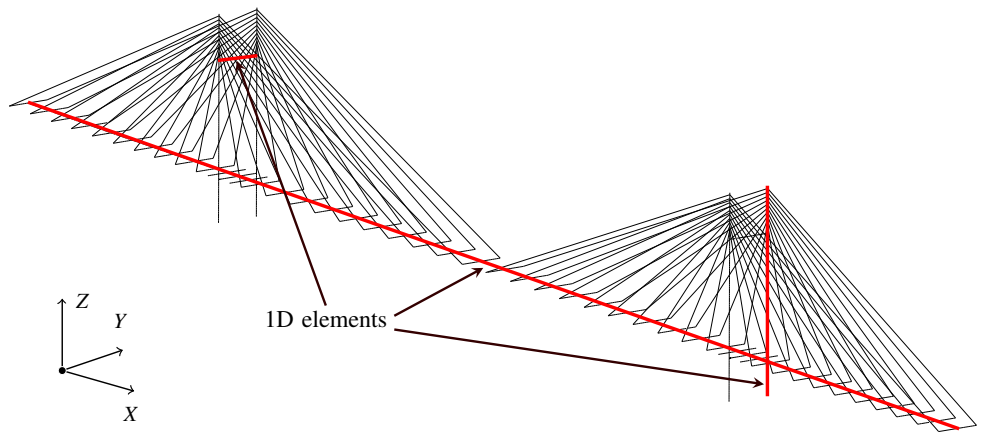

Figure 1: $\quad$ Some examples of 1D elements that can be found in a generic full DB: Job-1.odb Abaqus/Standard 6.13-2 Tue Jan 12 Biriziqge 291 prototype.

processing the MODESTO software is based on designing sequentially one by one these generic $1 \mathrm{D}$ elements.

The 1D elements in the reduced models are composed of a structural spine which emulates the stiffness of the prototype, and a set of cladding modules that represent the outside geometry and adjust the mass of the model, as it is depicted in figure 2. Consequently, the process can be divided into a first step
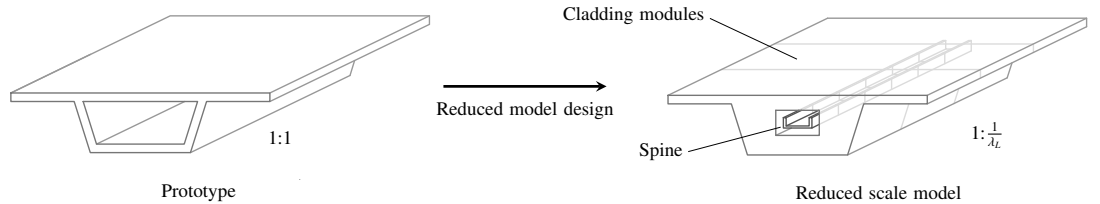

Figure 2: Conceptual scheme of the design of a 1D element of a full bridge deck model.

which consists on designing the stiffness properties of the spine and obtaining the dimensions of its cross-sections, and a second step on which the mass properties of the cladding modules are defined to emulate the mass properties of the prototype, after subtracting the mass properties of the spine. These steps are described below. 


\subsection{Stiffness properties design}

The main challenge of the design of the spine is to represent the stiffness properties of the prototype, which are variable along the length of the 1D element, by means of an element with uniform dimensions along the length of its segments. This is a design constraint due to constructive limitations. Thus, the stiffness properties design process is divided into two steps; a first step for defining the location of the segment with uniform properties along the spine, and a second step for obtaining the dimensions of each segments that reproduce the stiffness of the prototype. The first step is carried out heuristically depending on the location of the changes in the loads and stiffness properties of the prototype. The second steps aims to define the dimensions of the cross-sections of the segments defined in the first step. This is done from the properties of the prototype in the corresponding length of the segment. The rules for calculating the segment properties are based on the fundamental bending theory of beams, which establish the bending rotation $\theta_{y}$ as

$$
\theta_{y}=\frac{d^{2} z}{d x^{2}}=\frac{M_{y}(x)}{E I_{y}(x)}
$$

where $M_{y}$ is the bending moment, $E$ is the Young's modulus and $I_{y}$ is the inertia of the cross-section.

Besides, assuming that the loading $M(x)$ is uniform along the segment and that the Young's modulus is also uniform, eq. (2) can be written in terms of the corresponding length of the segment in the prototype $L_{\mathrm{p}}$ as

$$
\theta_{y, \mathrm{p}}=\int_{0}^{L_{\mathrm{p}}} \frac{M_{y, \mathrm{p}}(x)}{E_{\mathrm{p}} I_{y, \mathrm{p}}(x)} d x=\frac{M_{y, \mathrm{p}}}{E_{\mathrm{p}}} \int_{0}^{L_{\mathrm{p}}} \frac{1}{I_{y, \mathrm{p}}(x)} d x,
$$

where the subindex $p$ indicates prototype and $m$ stands for reduced model. It must be borne in mind that $I_{y, \mathrm{p}}(x)$ can be non uniform along the segment and that it the property to discretize. This property follows a continuous distribution along the equivalent segment length in the prototype, but is very common in the industry to define this continuous distribution by discrete values along the beams (for instance, in a finite element model). When this happens, eq. (3) can be expressed as a sum of discrete values instead of integrating the properties along the segment, as

$$
\begin{aligned}
\theta_{y, \mathrm{p}} & =\frac{M_{y, \mathrm{p}}}{E_{\mathrm{p}}} \int_{0}^{L_{\mathrm{p}}} \frac{1}{I_{y, \mathrm{p}}(x)} d x= \\
& =\frac{M_{y, \mathrm{p}}}{E_{\mathrm{p}}}\left[\int_{0}^{L_{1}} \frac{1}{I_{y, \mathrm{p}, 1}(x)}+\ldots+\int_{L_{n-1}}^{L_{n}} \frac{1}{I_{y, \mathrm{p}, n}(x)}\right]=\frac{M_{y, \mathrm{p}}}{E_{\mathrm{p}}} \sum_{l=1}^{n} \frac{L_{l}}{I_{y, \mathrm{p}, l}},
\end{aligned}
$$

where, $n$ are the number of discrete values that define $I_{y, \mathrm{p}}(x)$ along the equivalent length of the model's segment, $L_{\mathrm{p}}$ and $L_{l}$ are the lengths of the intervals were the properties are defined in the prototype. The model values can be obtained from the 
prototype ones applying the scale factors defined in eq. (1), as

$$
L_{m}=L_{p} \lambda_{L}, \quad M_{y, m}=M_{y, p} \lambda_{\mathrm{BM}}, \quad \text { and } \quad I_{y, m}=I_{y, p} \lambda_{\mathrm{EI}} .
$$

Therefore, eq. (4) can be written in terms of the reduced model properties $\left(\theta_{y, p}^{\mathrm{m}}\right)$ as

$$
\theta_{y, \mathrm{p}}^{\mathrm{m}}=\frac{M_{y, \mathrm{p}} \lambda_{\mathrm{BM}}}{E_{\mathrm{p}}} \sum_{l=1}^{n} \frac{L_{l} \lambda_{L}}{I_{y, \mathrm{p}, l} \lambda_{\mathrm{EI}}} .
$$

On the other hand, the deflection along a segment of the reduced model spine is given by

$$
\theta_{y, \mathrm{~m}}=\frac{M_{y, \mathrm{~m}}}{E_{\mathrm{m}}} \int_{0}^{L_{\mathrm{m}}} \frac{1}{I_{y, \mathrm{~m}}(x)} d x=\frac{M_{y, \mathrm{~m}} L_{\mathrm{m}}}{E_{\mathrm{m}} I_{y, \mathrm{~m}}}
$$

where $L_{m}$ is the length of the segment, and it has been considered that the segments of the spine have uniform stiffness properties along their length, as

$$
I_{y, \mathrm{~m}}(x)=I_{y, \mathrm{~m}} \text { and } E_{\mathrm{m}}(x)=E_{\mathrm{m}} \text {. }
$$

Hence, from eq. (6) and eq. (7) it is possible to establish the relation between the properties in the prototype and the reduced model as

$$
\theta_{y, \mathrm{~m}}=\theta_{y, \mathrm{p}}^{\mathrm{m}} \rightarrow \frac{L_{\mathrm{m}}}{E_{\mathrm{m}} I_{y, \mathrm{~m}}}=\frac{1}{E_{\mathrm{p}}} \sum_{l=1}^{n} \frac{L_{l} \lambda_{L}}{I_{y, \mathrm{p}, l} \lambda_{\mathrm{EI}}} \rightarrow I_{y, \mathrm{~m}}=\frac{E_{\mathrm{p}}}{E_{\mathrm{m}}} \frac{L_{\mathrm{m}}}{\sum_{l=1}^{n} \frac{L_{l} \lambda_{L}}{I_{y, \mathrm{~m}, l} \lambda_{\mathrm{EI}}}}
$$

where the sum of the lengths of the intervals $L_{l}$ is the total length of the segment $L_{\mathrm{m}}=\sum_{l=1}^{n} L_{l}$, and in the same way the torsional constant can be obtained from

$$
J_{x, \mathrm{~m}}=\frac{E_{\mathrm{m}}}{E_{\mathrm{m}}} \frac{L_{\mathrm{m}}}{\sum_{l=1}^{n} \frac{L_{l} \lambda_{L}}{J_{x, \mathrm{~m}, l} \lambda_{\mathrm{EI}}}} .
$$

where the term $\frac{E_{\mathrm{m}}}{\hat{E}_{\mathrm{m}}}$ takes into account the material change from the prototype to the reduced model.

\subsubsection{Calculus of the dimensions of the spine cross-sections}

With the relations established in eqs. (9) and (10), it is possible to obtain the stiffness properties of the spine. Thus, the last task is to define the cross-section geometry of the spine providing those stiffness properties. The channel section is a suitable section that allows to reproduce any combination of the bending inertias and torsional constants $\left(J_{X}, I_{Y}\right.$ and $\left.I_{Z}\right)$ by means of defining four dimensions $(b$, $d, b f$ and $d f$ ), as shown in figure 3 . The main drawback of this step in the process is to obtain the dimensions of every cross-section of all the spines of the full bridge model, and is here where the advantages of using the software tool presented in this work stand out. The definition of the four dimensions of each cross-section are obtained numerically by employing a gradient-based optimization algorithm 


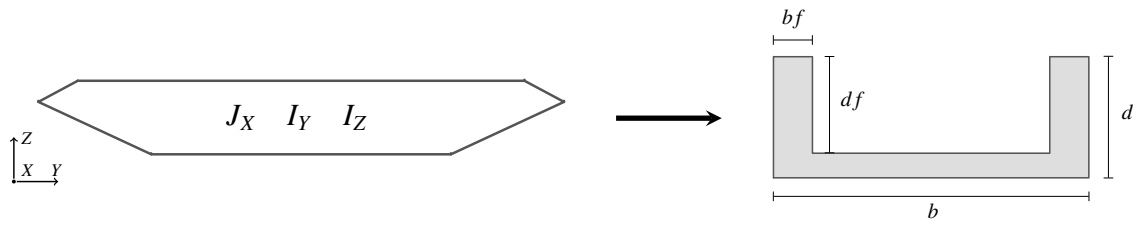

Figure 3: Simplification of the process of defining the reduced equivalent channel section.

$[16,17]$, formulating the objective function $f$ as

$$
f=\lambda_{J_{X}} \delta_{J_{X}}+\lambda_{I_{Y}} \delta_{I_{Y}}+\lambda_{I_{Z}} \delta_{I_{Z}}
$$

where $J_{X}$ is the torsional stiffness, $I_{Y}$ and $I_{Z}$ are the vertical and lateral bending stiffnesses, respectively, and $\delta_{I}$ is the relative error between the prototype and the reduced model properties of each stiffness, which are obtained from

$$
\delta_{I}=\frac{\left|I_{\mathrm{m}}-I_{\mathrm{p}}\right|}{I_{\mathrm{m}}} .
$$

In eq. (12) both stiffnesses are expressed in the same scale and the material change is considered. Besides, the relative errors are weighted by the weighting factor $\lambda_{I}$.

In this way, the dimensions of every cross-section is obtained sequentially with computer times in the order of seconds for the whole set of the 1D element, thanks to the sequential application of the optimization algorithm for each cross-section of the spine.

\subsection{Mass properties design}

After the design of the spine cross-sections in the reduced model, the next step is to determinate the mass properties of the cladding modules, which are the mass $M$ and the mass moment of inertia $I$. This is carried out by discretizing the length of the $1 \mathrm{D}$ element and distributing the mass properties of the prototype in the cladding modules after subtracting the mass provided by the spine. It must me noticed that structurally the mass properties of the cladding modules are transmitted to the spine by the clamping device that affix them to the spine, which means that the values of the mass properties of the cladding modules represent lumped masses and lumped mass moments of inertia added to the spine. Consequently, the cladding modules should be as short as constructively possible in order to improve the dynamic response of the reduced model thanks to a fine discretization of the mass along the mock-up. 


\subsection{Process automation}

This process can be automatized and the only decisions required by the user are the spine discretization, which is the definition of the segment lengths, and the mass discretization, which means establish the lengths of the cladding modules. These two tasks are carried out heuristically by the designer, and the rest of the process can be automatized. This is the basis of the software presented in this work, which in a user friendly way it is able to design the whole reduced models in a very short time.

\section{Software description and application example}

The automation of the process described above gives place to the MODESTO software presented in this paper, which was implemented in MATLAB code [18]. The main menu of the software is shown in figure 4, and is designed to work separately on the different $1 \mathrm{D}$ elements that compose a full bridge model, as described earlier (see figure 1). In this work, the deck of a generic cable-stayed

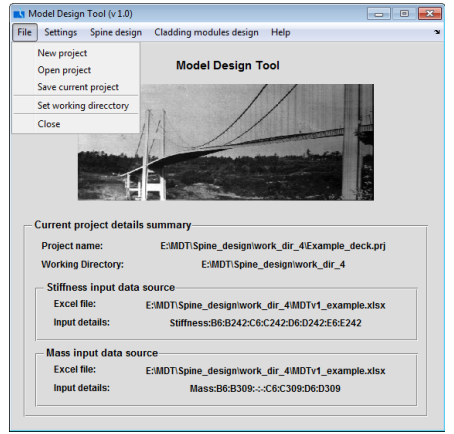

Figure 4: Main menu of MODESTO.

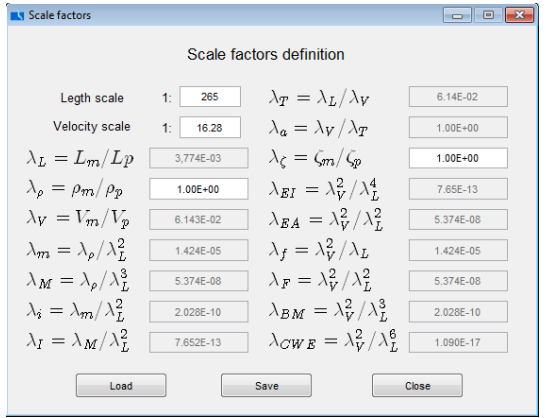

Figure 5: Scale factors form.

bridge with two towers and two piers is going to be used as an application example. The following sections describe the implementation of the process introduced before and the software interface to carry out the whole design.

\subsection{Settings definition}

Once the prototype is heuristically divided into $1 \mathrm{D}$ elements, the user can start working with one of them, as for instance, the deck of the bridge. The first task is to provide the length scale factor $\lambda_{L}$, so the software calculates all the scale factors involved in the design, following the expressions shown in eq. (1), as shown in figure 5. Besides, in order to establish the material change relations, such as $\frac{E_{\mathrm{p}}}{E_{\mathrm{m}}}$, the material properties form has to be filled in, as it can be seen in figure 6 . 


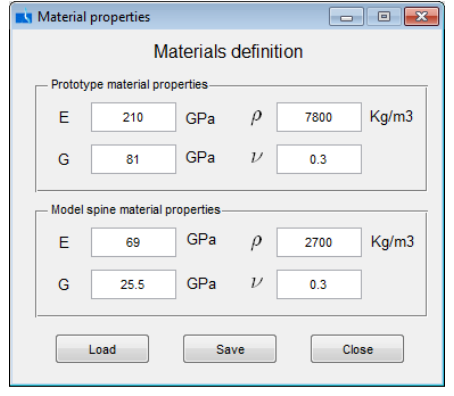

Figure 6: Material properties form.

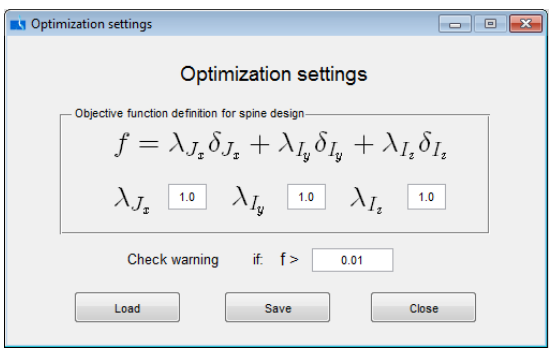

Figure 7: Optimization settings form.

The last required data is the definition of the objective function (eq. (11)) to obtain the corresponding dimensions of the spine, which is carried out in the form shown in figure 7 . The weighting factors of the stiffness properties $\lambda_{I}$ are also required, as well as a convergence warning value. This warning value is a threshold that indicates when the convergence of the optimization algorithm is not achieved for a particular cross-section set of stiffness properties, which can help the user to detect errors in the data provided to the program.

\subsection{Spine design}

The next step consists on providing the prototype's stiffness properties for every cross-section of the 1D elements. Once this is done, all the information required for the spine design is already provided, and the only remaining task is to define the length and location of the segments along the $1 \mathrm{D}$ element. This is done in the form shown in figure 8 , which, employing this information, calculates the dimensions of each cross-section of the spine by applying the optimization algorithm as many times as segments are defined. The result for this example is presented in figure 9, where the dimensions of the spine along the length of the deck are shown. It can be seen that, as the stiffness properties values modeling the deck are increased in the vicinity of the towers and piers, as shown in figure 8 , the dimensions of the cross-section of the spine are also increased in those particular locations, as shown in figure 9. On the other hand, the cross-section dimensions along the main span are mostly uniform.

\subsection{Cladding modules design}

Once the spine dimensions are obtained and consequently the mass properties of the spine can be determined, the identification of the mass properties of the cladding modules can be conducted. This is carried out by filling up the form shown in figure 10. After the mass properties of the prototype are provided to the software, the user can define the lengths of the cladding modules that will 


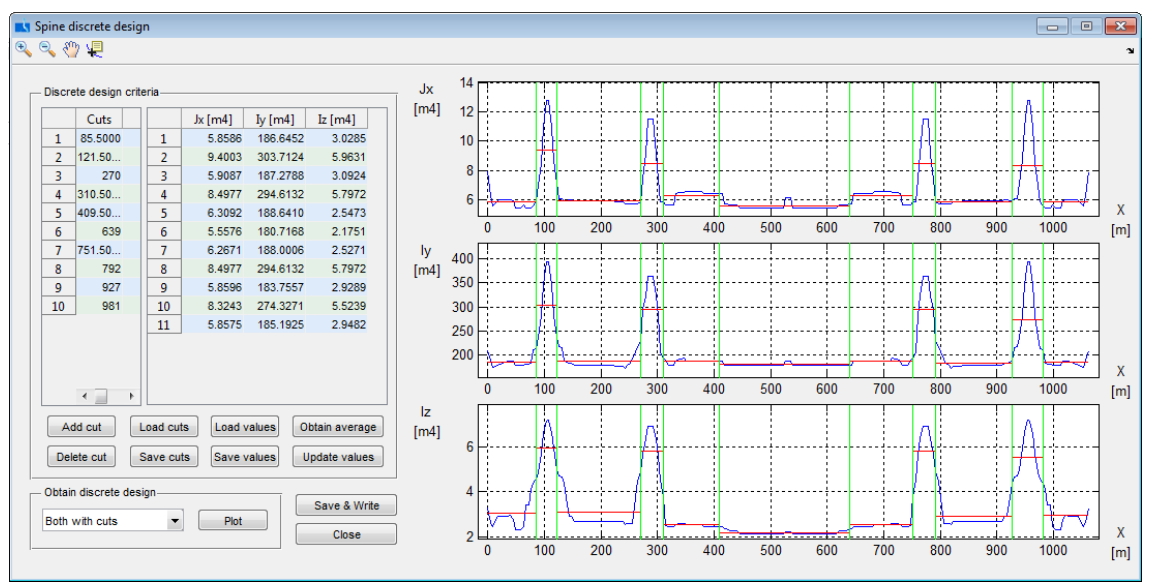

Figure 8: Spine design form.
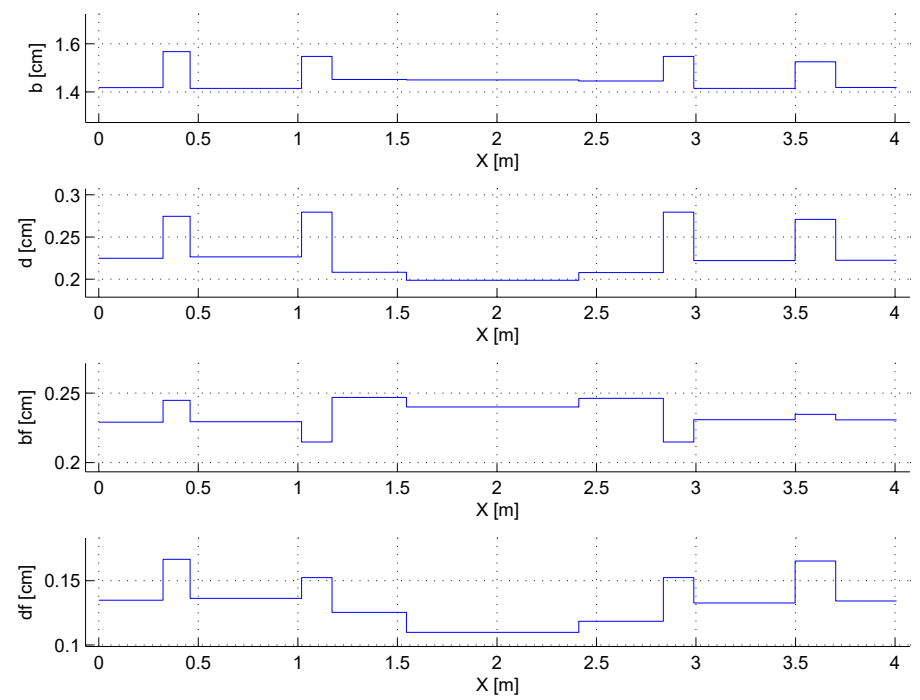

Figure 9: Spine dimensions of the reduce model 1D element along its length.

compose the reduced model. The software calculates the mass that belongs to each cladding module by associating the corresponding mass properties of each length in the prototype and subtracting the mass properties of the snipe. It can be seen in figure 10 the mass properties of the spine in black, the mass properties of the prototype in blue, the discretized mass properties of the prototype in red and the mass properties of the cladding modules after subtracting the values of the spine in pink. It must be borne in mind that the cladding modules have to be as short 


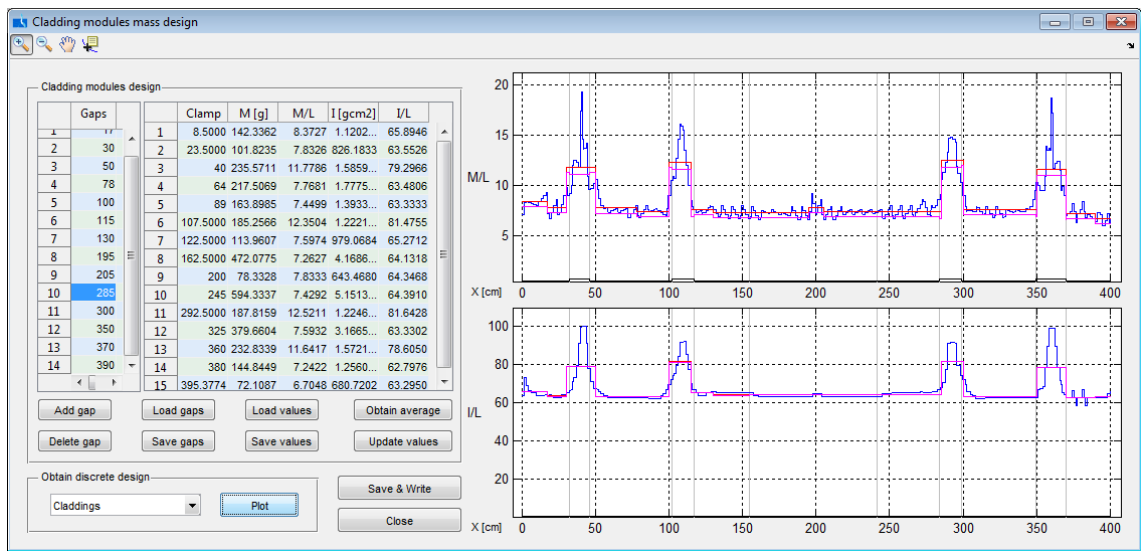

Figure 10: Cladding modules design form.

as constructively feasible in order to avoid excessive values in the lumped masses that the cladding modules represent in the spine, aiming to increase the number of lumped masses and decrease the influence of each one.

Thus, in the presented fashion, the spine dimensions and the cladding modules mass properties are defined in an accurate way, reducing possible human errors, and in a very short working time, which is expected to be in the order of minutes.

The feasibility of the described approach is verified by comparing the differences in the natural frequencies of the prototype and the one of the reduced model after applying the corresponding scale factors. In the same way, the modal shapes and the generalized masses are also contrasted, as well as any other dynamic response of interest. It has been found that the errors present in all the dynamic properties of the reduced model employed as application example in this work are very small and consequently the resulting design is acceptable.

\section{Conclusions}

This papers presents a short description of the process of designing a full bridge reduced model for wind tunnel aeroelastic tests. First, the main rules of model design and similitude requirements are described and the scale factors that these laws give place to are presented. Second, the process for obtaining the stiffness properties of the reduced model are outlined, and an automatic way for obtaining the dimensions of the cross-section of the spine employing optimization algorithms is presented. Third, the cladding modules design process is also commented.

Furthermore, an implementation of all these process is presented and tested with an application example of a generic cable-stayed bridge deck. It allows the user to 
obtain an accurate design avoiding human errors in the process in a very reduced working time, simplifying the whole wind tunnel test modeling work.

\section{Acknowledgements}

The research leading to these results has received funding from the Spanish Minister of Economy and Competitiveness (MINECO) with reference BIA201341965-P and the Fundación Pedro Barrié de la Maza and the Universidade da Coruña. The authors fully acknowledge the support received.

\section{References}

[1] Davenport, A.G. \& Isyumov, N., The application of the boundary layer wind tunnel to the prediction of wind loading. International Research Seminar: Wind Effects on Buildings and Structures, 1967.

[2] Isyumov, N., Tschanz, T. \& Davenport, A.G., Wind action on a cable-stayed bridge. $3^{\text {th }}$ US National Conference on Wind Engineering, Gainesville, Florida, USA., 1978.

[3] Irwin, P.A., The role of wind tunnel modeling in the prediction of wind effects on bridges. Proceedings of the International Symposium on Advances in Bridge Aerodynamics., 1998.

[4] Simiu, E. \& Scanlan, R.H., Wind effects on structures. John Wiley \& Sons, 1996.

[5] Xu, Y.L., Wind effects on cable-supported bridges. John Wiley \& Sons, Singapore, 2013.

[6] Davenport, A.G. \& King, J.P.C., The influence of topography on the dynamic wind loading of long span bridges. Journal of Wind Engineering and Industrial Aerodynamics, 36, pp. 1373-1382, 1990.

[7] Davenport, A.G., The response of suspension bridges to wind action. International Research Seminar on Safety of Structures, 1977.

[8] Davidson, P.A., Turbulence. An introduction for scientists and engineers, second edition. Oxford University Press, Oxford, UK., 2015.

[9] Larose, G.L., Davenport, A.G. \& King, J.P.C., Wind effects on long span bridges: Consistency of wind tunnel results. Journal of Wind Engineering and Industrial Aerodynamics, 42, pp. 1191-1202, 1992.

[10] Zasso, A., Cigada, A. \& Negri, S., Flutter derivatives identification through full bridge aeroelastic model transfer function analysis. Journal of Wind Engineering and Industrial Aerodynamics, 60, pp. 17-33, 1996.

[11] Terrés-Nícoli, J.M., Kong, L., King, J.P.C. \& Mans, C., Study of the wind effects on a $384 \mathrm{~m}$ concrete arch span bridge during different construction phases. $37^{\text {th }}$ IASBE Symposium on Engineering for Progress, Nature and People; Madrid, Spain., 2014.

[12] Davenport, A.G., Isyumov, N., Fader, D.J. \& Bowen, C.F.P., A study of wind 
action on a suspension bridge during erection and on completion. BLWTL3-69, University of Western Ontario, 1969.

[13] Isyumov, N., Tschanz, T. \& Davenport, A.G., A study of wind action for the Weirton-Steubenville cable-stayed bridge. BLWTL-SS1-77, University of Western Ontario, 1977.

[14] Barlow, B., Rae, W.H. \& Pope, A., Low-speed wind tunnel testing. WileyInterscience, 1999.

[15] Dyrbye, C. \& Hansen, S.O., Wind loads on structures. John Wiley \& Sons Inc., 1999.

[16] Hernández, S. \& Fontán, A.N., Practical applications of design optimization. WIT Press, 2002.

[17] Arora, J.S., Introduction to optimum design. Elsevier/Academic Press, 3rd edition, 2011.

[18] Mathworks, MATLAB 2013a documentation, 2013. 